Doug Geisler, Eva K. Grebel, and Dante Minniti, eds.

\title{
Deep Wide-Field Photometry of the NGC 5128 Globular Cluster System: A Progress Report
}

\author{
G. L. H. Harris \\ University of Waterloo, Waterloo, Ontario, Canada \\ D. Geisler \\ Universidad de Concepción, Concepción, Chile \\ W. E. Harris \\ McMaster University, Hamilton, Ontario, Canada
}

J. E. Hesser

HIA/NRC, Victoria, British Columbia, Canada

\begin{abstract}
We have obtained $C M R$ photometry for a roughly $1^{\circ}$ square region centered on NGC 5128. Preliminary results indicate that the limiting magnitude of the images is $\gtrsim 1$ magnitude fainter than the peak of the globular cluster luminosity function (GCLF) at $R \simeq 21$.
\end{abstract}

\section{Introduction}

At a distance of $\sim 4 \mathrm{Mpc}$, NGC 5128 is the nearest giant elliptical galaxy, providing us with a unique opportunity to study detailed properties of its stellar halo in both field stars and globular clusters. However, this proximity also means that the galaxy extends over $\geq 1^{\circ}$ in the sky, making imaging of the whole system impossible until recently. As a result, our knowledge of NGC 5128's globular cluster system (GCS) has been based mainly on the photographic survey work of H. Harris et al. (1984) and the $C M T_{1} T_{2}$ CCD photometry of a small sample (G. Harris et al. 1992) of its probably $>1500$ clusters. With the availability of the wide field BTC camera on the $4 \mathrm{~m}$ telescope of the Cerro Tololo InterAmerican Observatory it became possible to redress this situation.

\section{Present Results}

\subsection{Observations and Analysis}

During observing runs in May 1999 and May 2000, we obtained images in $C M R$ for nine partially overlapping fields centered on NGC 5128 and extending to $\sim 35^{\prime}$ from the galaxy center. The overlap in exposures served to fill in the gaps between chips in the BTC camera and provide an internal calibration of the photometry, both chip to chip and night to night. Exposure times were 
2000s in $C$ and 500s in $M$ and $R$; because many of the brightest clusters were saturated in these images, additional short exposures of 100 s in $M$ and $R$ were obtained for each field. With the overlapping exposures we expect to have $\geq 2$ observations of $\geq 30 \%$ of the detected candidates.

The images were analyzed using Daophot within IRAF; cluster candidates with large photometric errors and poor image quality were deleted from the sample. At this stage the photometric calibration is rudimentary and based on comparison with the aperture photometry of G. Harris et al. (1992).

\subsection{Preliminary Results}

We currently have results for three $15^{\prime} \times 15^{\prime}$ frames from indivudual chips within the $4 \times 4$ BTC camera field. These are position 3-2 located $\sim 10^{\prime} \mathrm{SW}$ of the galaxy center, position 3-3 at $\sim 25^{\prime} \mathrm{SW}$, and position 7-4 at $\sim 45^{\prime} \mathrm{SW}$. In position 3-2 the limiting magnitude of the photometry is $R_{\sim}^{>22}$ and the GCLF peak is distinct at the expected value of $R \sim 21$. The color distribution of the probable clusters is clearly bimodal, with the majority of the population in the red (metal-rich?) peak. The cluster population is present in position 3-3, but far fewer cluster candidates are present at this greater distance from the galactic center. The data for position 7-4 are dominated by field stars and we expect that the outer fields can be used to evaluate the background in the direction of NGC 5128. The dominant population at $R_{\sim}^{>22}$ appears to be due to contamination from faint stars of the Milky Way which are present in large numbers because of the low Galactic latitude of NGC 5128. Consequently, the detection of clusters in NGC 5128 by purely photometric means will be limited to $\geq 1$ magnitude fainter than the GCLF turnover. Nonetheless, we anticipate that these images will provide a candidate list of $\sim 1000$ globular clusters from which systemic properties such as spatial distribution, luminosity function, and color/metallicity distribution can be determined. The list will also provide a basis for more detailed studies of cluster abundances and, possibly, ages. Finally, we will be better able to compare the NGC 5128 globular cluster system with its bright, old halo stars which appear to have had a significantly different formation and enrichment history (Harris et al 1999; Harris and Harris 2000).

\section{References}

Harris, G. L. H., Geisler, D., Harris, H. C., \& Hesser, J. E. 1992, AJ, 104, 613

Harris, G. L. H., Harris, W. E., \& Poole, G. B. 1999, AJ, 117, 855

Harris, G. L. H. \& Harris, W. E. 2000, AJ, 120, 2423

Harris, H. C., Harris, G. L. H., Hesser, J. E., \& MacGillivray, H. T. 1984, ApJ, 287,185 

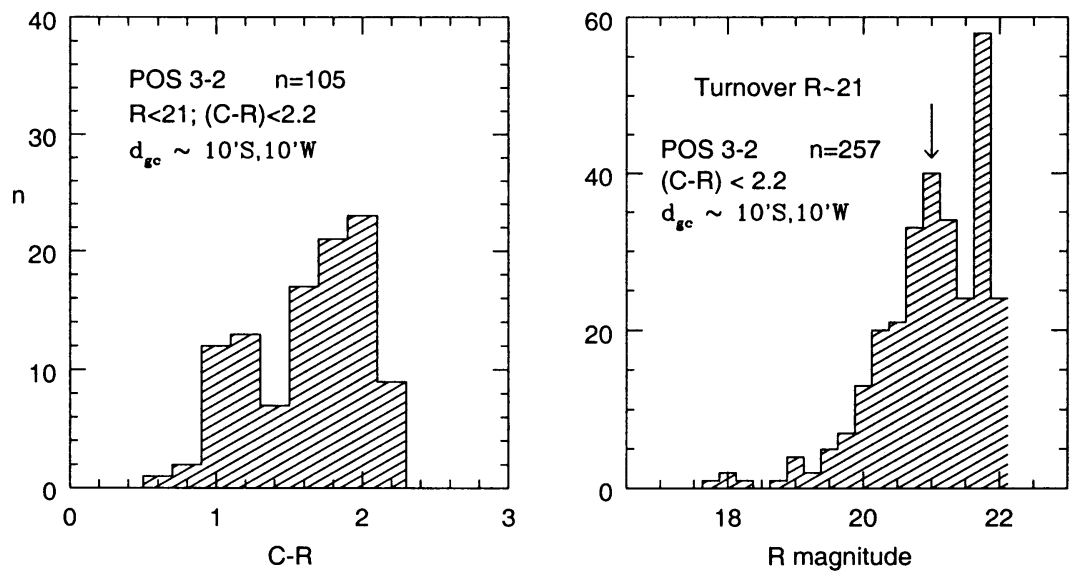

Figure 1. Preliminary color (left) and luminosity (right) distributions for probable clusters in Position 3-2. The color (metallicity) distribution is clearly bimodal; the luminosity distribution has a well defined peak near the expected turnover of $R \simeq 21$. 